\title{
Mobility models for next generation wireless mesh network
}

\author{
Tsehay Admassu Assegie ${ }^{1}$, Tamilarasi Suresh ${ }^{2}$, R. Subhashni ${ }^{3}$, Deepika $\mathbf{M}^{\mathbf{4}}$ \\ ${ }^{1}$ Department of Computer Science, Faculty of Computing Technology, Aksum Institute of Technology, Aksum \\ University, Aksum, Ethiopia \\ ${ }^{2,4}$ Department of Information Technology St. Peter's Institute of Higher Education and Research, \\ Chennai, India \\ ${ }^{3}$ Department of Computer Science and Applications, St. Peter's Institute of Higher Education and Research, Chennai,
} India

\begin{tabular}{l}
\hline Article Info \\
\hline Article history: \\
Received May 1, 2019 \\
Revised Feb 21, 2021 \\
Accepted Mar 3, 2021 \\
\hline
\end{tabular}

\section{Keywords:}

Gauss Marko's mobility model

Node mobility

Performance evaluation

Software defined network

Wireless network emulation

\begin{abstract}
Wireless mesh network (WMN) is a new trend in wireless communication promising greater flexibility, reliability, and performance over traditional wireless local area network (WLAN). Test bed analysis and emulation plays an essential role in valuation of software defined wireless network and node mobility is the prominent feature of next generation software defined wireless network. In this study, the mobility models employed for moving mobile stations in software defined wireless network are explored. Moreover, the importance of mobility model within software defined wireless mesh network for enhancing the performance through handover-based load balancing is analyzed. The mobility models for the next generation software defined wireless network are explored. Furthermore, we have presented the mobility models in the mininet-Wi-Fi test bed, and evaluated the performance of Gauss Marko's mobility model.
\end{abstract}

This is an open access article under the CC BY-SA license.

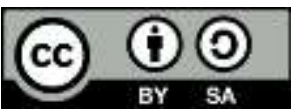

\section{Corresponding Author:}

Tsehay Admassu Assegie

Department of Computer Science

Aksum University

Axum, Ethiopia

Email: tsehayadmassu2006@gmail.com

\section{INTRODUCTION}

The pattern of mobility of nodes in the network considerably affects the performance of software defined wireless network [1-25]. In wireless local area network (WLAN), the nodes are mostly mobile devices, such as laptops, smart phones and tablets, typically used by the people to get access to wireless local area network. However, the wireless local area network consists of fixed access points (APs) and a number of mobile devices.

The access point usually connects so many mobile devices, such as university students in a campus and so on. The human mobility plays a major role on the wireless local area network performance evaluation. In wireless local area network, node mobility is characterized by the trajectory, location of the mobile nodes and analysis of migration patterns between Aps in the network [6-12]. When users migrate or move from one location to another location, then they de associate and association to APs in the network. Another important reflection about wireless local area network user mobility is that the mobility of nodes is often intermittent in the time domain. In other words, a user can be connected with an access point, A at time t1, then dis-connected from access point, $\mathrm{A}$, and later on (at time $\mathrm{t} 2 \geq \mathrm{t} 1$ ) "re-connected to access point $\mathrm{A}$ " in the network connects to a different access point, say B. This form of mobility pattern is common, for example, in 
a campus network, where students move from, say, classroom to library, and turn off their laptops while moving from one location to the other.

The next generation networks, such as the wireless sensor network and software defined wireless network are connected in mesh topology, in contrast to the traditional wireless network, which is usually connected in the point-to-point topology [13]. The wireless sensor network and software defined wireless network are greatly affected by node mobility. Mobility plays a leading role on the performance of such networks. This study focuses on explaining the extensive research works devoted to the next generation wireless network, namely the software defined wireless network mobility models. The mobility models have a major goal of mimicking the human behavior, animal behavior or even vehicular mobility $[14,15]$.

\section{RELATED WORKS}

In this section, the prior research works, related to node mobility models for the next generation software defined network and wireless sensor network, are discussed. Some of the research works on mobility models for the next generation network are discussed in [16-20]. The traditional wireless local area network is based on the point-to-point or point-to-multi-point topologies [16]. However, recently, the new networking topology, the mesh topology has emerged in the wireless network systems. The mesh topology has become not as an option but it is a necessity in the next generation wireless networks, such as the Wireless Sensor Networks, where nodes are required to transmit their data with peering devices. In such scenarios, the traditional topology will not enable the nodes to transmit their data faster with the nearby nodes. Mobility management deals with two issues, the location management and handover management. Location management is task of monitoring the location of every node in the wireless network and handover management deals with how nodes can be handed over from an access point to another access point. The handover decision is made based on the signal strength or the load of access points.

Mobility model describes the mathematical model used to describe the motion of mobile nodes in a wireless network. Gauss Markov's mobility model, as its name suggest is a Gaussian based mobility model [17]. The random Way Point is a simplest mobility model widely used in evaluation of MANE protocols [18]. This is due to its simplicity in implementation. This model chooses a random destination and constant speed, which the node uses to, reaches its destination. The parameters required for this model are, the maximum and minimum speed and the pause time.

Mobility models have a great influence on the performance of wireless mesh networks [19]. This is because node movement may overload some Access points in the network while other access points are free in the network. The recent mobility model widely used in wireless network for real network mobile node mobility emulation which is supported by the mininet-Wi-Fi, the software defined wireless network is the Gauss Markov's mobility model [20].

\subsection{Wireless network mobility models}

A mobility model is a method for simulating motions of mobile nodes in wireless networks used primarily for simulation. Mininet-WiFi supports the following mobility models:

- RandomWalk,

- TruncatedLevyWalk,

- RandomDirection,

- RandomWayPoint,

- GaussMarkov,

- ReferencePoint and

- TimeVariantCommunity.

\subsubsection{Gauss Markov's mobility model}

This section discusses Gauss Markov's mobility model; in detail as the focus of this study is the Gauss Markov's mobility model. This model is usually applied to position nodes in high mobility network. This model employs two parameters [4], speed and direction. Each node in the network, updates its speed and direction in the pause time. Moreover, the Gauss Markov's model provides better authentic model for mobile nodes. Figure 1, shows the schematic diagram of node movement when the Gauss Markov's model used in node mobility, with parameters max_x=200, max_y=200, speed=20 and time $=1$ ). Figure 1, demonstrates the pseudo code for creating Gauss markov's mobility model in software defined wireless network emulation tool, mininet Wi-Fi. 
In Figure 2, the Gauss Markov's mobility model is demonstrated. Three mobile nodes, namely, Station-1, Station_3, and Station_3 are created for emulating Gauss Markov's mobility model in a software defined wireless local area network covering 300 by 300 meters square area.

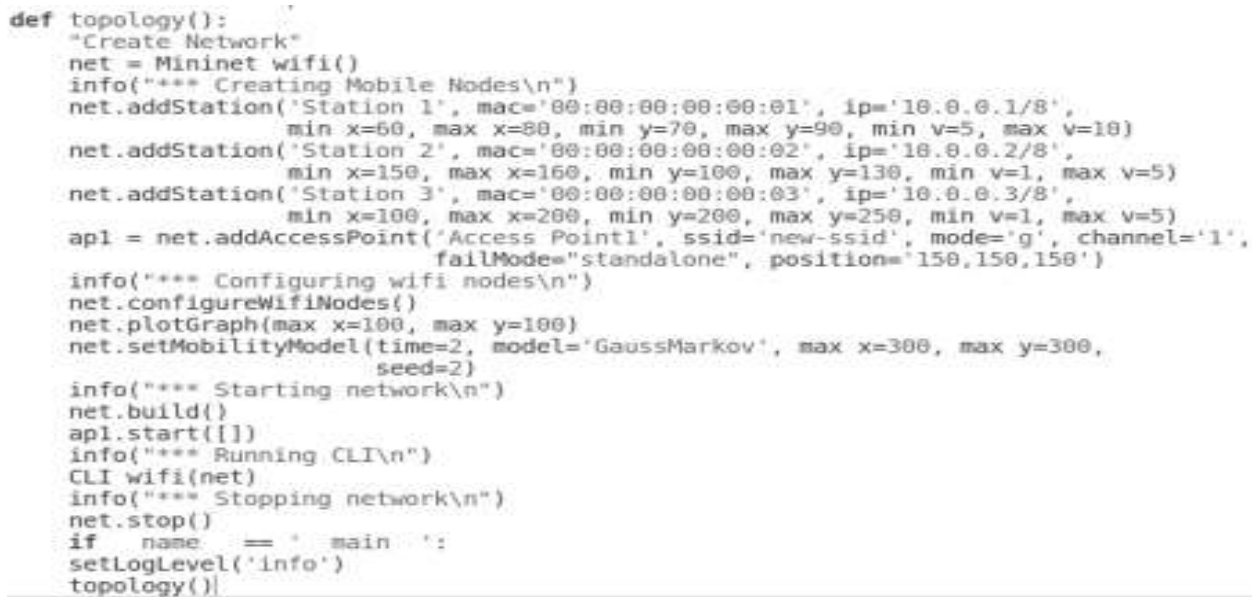

Figure 1. Pseudo code of Gauss Markov's mobility model

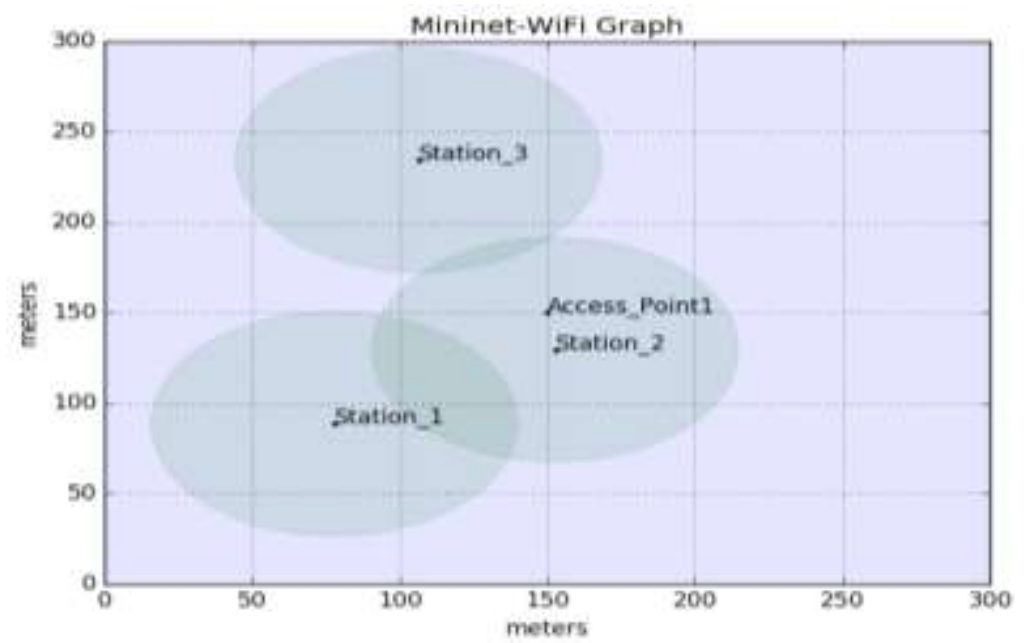

Figure 2. Gauss Markov's node mobility model, mininet-Wi-Fi graph

\section{RESEARCH METHOD}

In this research, mininet-Wi-Fi emulation tool is employed to simulate the Gauss Markov's mobility model in software defined wireless mesh network. The Gauss Markov's mobility model is one of the mobility model for software defined wireless network to measure the transmission control protocol (TCP) traffic between mobile stations in the software defined wireless network emulation environment, we employed IPERF, a TCP traffic bandwidth measurement tool.

\section{RESULTS AND DICUSSIONS}

In this section, we will create a scenario where one station moves about in a virtual space, and where it changes which access point it connects to, based on which access point is the closest to it. The test-bed based emulations were carried out using the mininet-wifi emulation tool. For the emulation of Gauss Markov's mobility model we have created the mesh network topology consisting of three nodes as shown in Figure 3 using the mininet-Wi-Fi emulation tool. Figure 3 shows wireless local area network mesh topology. 


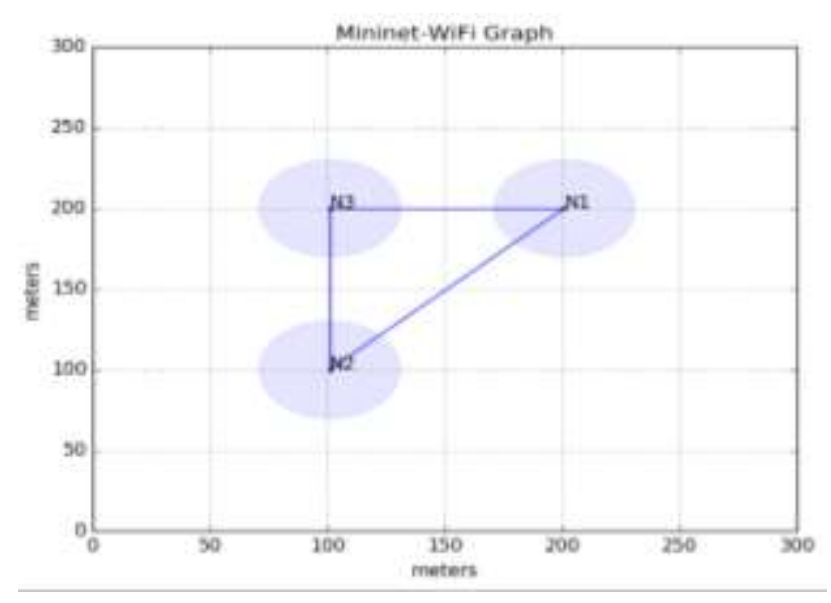

Figure 3. Mesh topology, with three nodes

\subsection{Mobility based handover}

To create the scenario where stations in the wireless network will be mobile, we have set the range of the access points. In our test-bed environment we have used the strongest signal first (ssf) as the Association Control method. The range of each access point is employed to analyze where handoffs occur between access points and which stations may connect to which access points.

Mininet-WiFi can also automatically move stations around based on predefined mobility models. The GaussMarkov, for example is used to move a station around in an area 300 meters by 300 meters with a minimum velocity of 0.1 meters per second and a maximum velocity of 0.2 meters per second, using the python scrip shown in (1).

net. startMobility (startTime $=0$, model='GaussMarkov', $\max \_\mathrm{x}=300, \max \_\mathrm{y}=300, \min \_v=0.1$, max_v=0.2).

Figure 4 shows how Mininet-WiFi implements scenarios with mobile station that hand off between access points. Figure 4 (a) shows mobile station originally connected with access point (AP1) and Figure 4 (b) shows the hand off station-1 to access point (AP2). Mininet-WiFi will automatically connect and disconnect stations to and from access points based on either calculated signal strength or load level.

net. startMobility (startTime $=0$, model='GaussMarkov', max_x=140, max_y=140, min _ v=0.7, max_v=0.9, AC='ssf')

The hand off is determined based on strength of the signal, strongest signla first (ssf), association method is used in the above scenario. To verify that if the sation Sta1 is connected to access point AP1, we can use the following commands shown in (3) and (4).

Before handoff mininet-wifi> sta1 iw dev sta1-wlan0 link

Connected to 02:00:00:00:03:00 (on sta1-wlan0) SSID: ssid_ap2 After hand off mininet-wifi> sta1 iw dev sta1-wlan0 link

Connected to 02:00:00:00:04:00 (on sta1-wlan0) SSID: ssid_ap1

\subsection{TCP traffic performance}

The performance of transmission control protocol (TCP) traffic between mobile stations is measured using IPERF with Gauss Markov's mobility model in Mininet-Wifi test bed environment is shown in Figure 5. As demonstrated in Figure 5, the performance of software defined wireless mesh network improves with handover employing the Gauss Markov's mobility model. The mobility model crucial for moving mobile nodes from overloaded wireless access point to the less overloaded access points, providing load balancing and ultimately improves the performance of the network. 


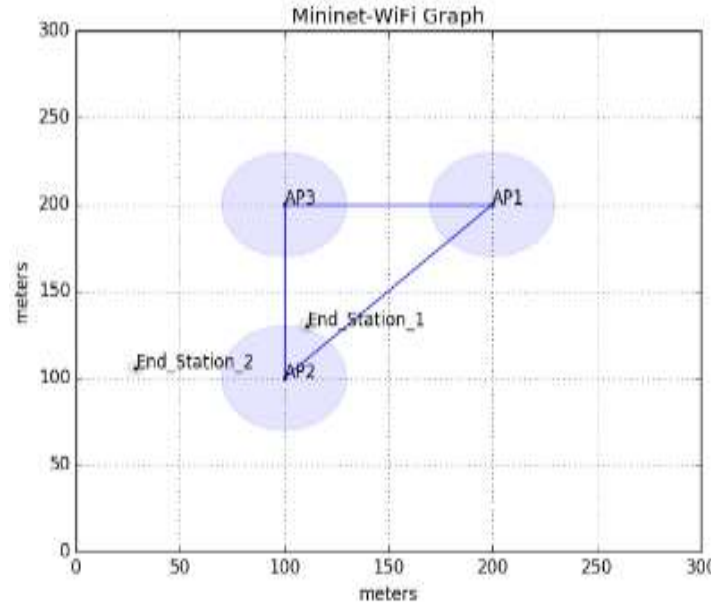

(a)

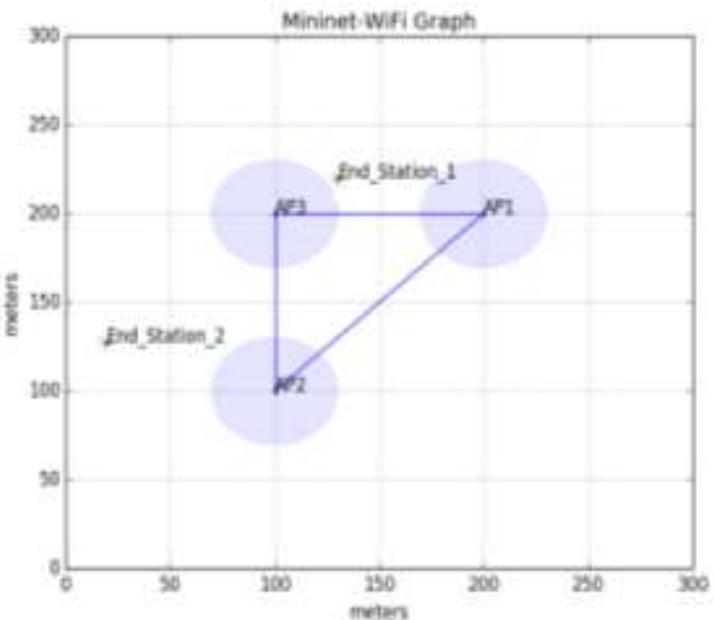

(b)

Figure 4. Mobility model in Mininet-Wi-Fi; (a) mobile nodes, Mesh Aps and (b) signal strength-based hand off

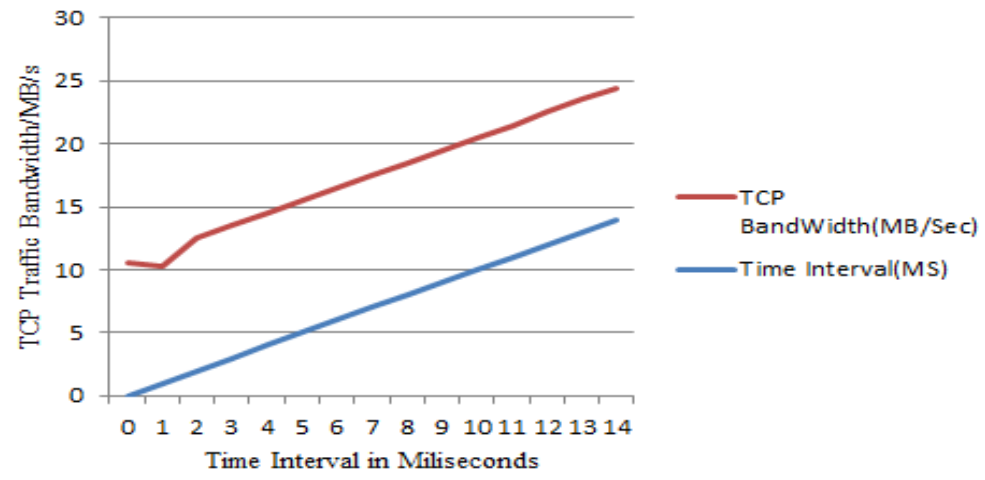

Figure 5. TCP traffic performance

\section{CONCLUSION}

Software defined wireless network (SDWN) is an emerging wireless network. In software defined wireless network, nodes are required to form a full mesh topology to enable communication between the nodes such as transmission of data between the nodes. In this study, we have emulated software defined wireless network using mininet-Wi-Fi and explored the Gaussian mobility model which is widely employed in software defined wireless network, to analyze and define the features of user mobility from one access point to the other access point. Experimental result appears to prove that the mobility model has a great influence on the network performance. Overall, load balancing handover, approach with mobility model improve the performance of software defined wireless network.

\section{REFERENCES}

[1] Meyanand R., Ramya Dorai D., "Analysis of Mobility Management in Wireless Mesh Networks using Random Walk Model," International Journal of P2P Network Trends and Technology (IJPTT), vol. 1, no. 2, pp. 17-23, 2011.

[2] Andrea Ribeiro, Rute C. Sofia, “A Survey on Mobility Models for Wireless Networks," UMM: User-centric Mobility Management, 2011.

[3] E. R. Cavalcanti, M. A. Spohn, "On the Interactions between Mobility Models and Metrics in Mobile ad hoc Networks," International Journal of Networks and Communications, vol. 3, no. 3, pp. 63-80, 2013, doi: 10.5923/j.ijnc.20130303.01.

[4] D. Chenghao, "An Improved Routing Protocol Based on Gauss-Markov Model In Ad Hoc Networks Utilizing Prediction of Link Quality," Proceedings of the 34th Chinese Control Conference, July 28-30, 2015, doi: 10.1109/ChiCC.2015.7260664. 
[5] N. A. Khan, F. Ahmad, S. A. Hussain, S. A. Khan, "Modeling and Simulation of CommunityMobility Model for Next Generation Wireless Networks using Coloured Petri Nets," IEEE 14th International Conference on Machine Learning and Applications, 2015, doi: 10.1109/ICMLA.2015.110.

[6] D. Towsley, "Mobility Models for Wireless Networks: Challenges, Pitfalls, and Successes," 22nd Workshop on Principles of Advanced and Distributed Simulation, IEEE 2008, doi: 10.1109/PADS.2008.28.

[7] X. Ge, J. Ye, Y. Yang, Q. Li, "User Mobility Evaluation for 5G Small Cell Networks Based on Individual Mobility Model," IEEE Journal on Selected Areas in Communications, vol. 34, no. 3, pp. 581-541, 2016, doi: 10.1109/JSAC.2016.2525439.

[8] I. T. Haque, N. Abu-Ghazaleh, "Wireless Software Defined Networking: A Survey and Taxonomy," Communications Surveys \& Tutorials, vol. 18, no. 4, pp. 1-1, 2016, doi: 10.1109/COMST.2016.2571118.

[9] T. A. Assegie, P. S. Nair, "Performance analysis of emulated software defined wireless network," Indonesian Journal of Electrical Engineering and Computer Science, vol. 16, no. 1, pp. 311-318, October 2019, doi: 10.11591/ijeecs.v16.i1.pp311-318.

[10] T. A. Assegie, P. S. Nair, "The performance of Gauss Markov's mobility model in emulated software defined wireless mesh network," Indonesian Journal of Electrical Engineering and Computer Science, vol. 18, no. 1, pp. 428-433, April 2020, doi: 10.11591/ijeecs.v18.i1.pp428-433.

[11] T. A. Assegie, Bizuneh H. D., "Improving network performance with an integrated priority queue and weighted fair queue scheduling," Indonesian Journal of Electrical Engineering and Computer Science, vol. 19, no. 1, pp. 241-247, July 2020, doi: 10.11591/ijeecs.v19.i1.pp241-247.

[12] F. Pakzad, S. Layeghyt, M. Portmann, "Evaluation of Mininet-WiFi Integration via ns-3," In 26th International Telecommunication Networks and Applications Conference, IEEE, 2016, doi: 10.1109/ATNAC.2016.7878816.

[13] S. M. Shamim, M. B. Alam Miah, A. Sarker, A. N. Bahar, A. Sarker, "Simulation of Minimum Path Estimation in Software Defined Networking Using Mininet Emulator," British Journal of Mathematics \& Computer Science, vol. 21, no. 3, pp. 1-8, 2017, doi: 10.9734/BJMCS/2017/30609.

[14] L. L. Zulu, K. A. Ogudo, P. O. Umenne, "Simulating Software Defined Networking Using Mininet to Optimize Host Communication in a Realistic Programmable Network," 2018 International Conference on Advances in Big Data, Computing and Data Communication Systems (icABCD), 2018, doi: 10.1109/ICABCD.2018.8465433.

[15] T. A. Assegie, "Software defined network emulation with OpenFlow protocol," International Journal of Advances in Applied Sciences (IJAAS), vol. 9, no. 1, pp. 70-76, March 2020, doi: 10.11591/ijaas.v9.i1.pp70-76.

[16] H. T. Zaw, A. H. Maw, "Traffic management with elephant flow detection in software-defined networks (SDN)," International Journal of Electrical and Computer Engineering (IJECE), vol. 9, no. 4, pp. 3203-3211, August 2019, doi: 10.11591/ijece.v9i4.pp3203-3211.

[17] H. Sounni, N. El kamoun, F. Lakrami, “A new SDN-based load balancing algorithm for IoT devices,” Indonesian Journal of Electrical Engineering and Computer Science, vol. 21, no. 2, pp. 12029-1217, February 2021, doi: 10.11591/ijeecs.v21.i2.pp1209-1217.

[18] T. A. Assegie, P. S. Nair, "Comparative Study on Methods Used in Prevention and Detection Against Address Resolution Protocol Spoofing Attack," Journal of Theoretical and Applied Information Technology, vol. 97, no. 16, August, 2019.

[19] F. A. Kamarudin, M. N. Megat Mohamed Noor, F. Ali, "A comparative study for bandwidth on demand using ONOS Reactive and Intent forwarding," Indonesian Journal of Electrical Engineering and Computer Science, vol. 17, no. 3, pp. 1410-1421, March 2020, doi: 10.11591/ijeecs.v17.i3.pp1410-1421.

[20] J. Xu, Y. Zhao, X. Zhu, "Mobility Model Based Handover Algorithm in LTE-Advanced," International Conference on Natural Computation, IEEE, 2014, doi: 10.1109/ICNC.2014.6975840.

[21] Y. C. Lai, A. Ali, M. S. Hossain, Y. D. Lin, "Performance modeling and analysis of TCP and UDP flows over software defined networks," Journal of Network and Computer Applications, vol. 130, pp. 76-88, 2019, doi: 0.1016/j.jnca.2019.01.010.

[22] T. A. Assegie, P. S. Nair, "A review on software defined network security risks and challenges," TELKOMNIKA Telecommunication, Computing, Electronics and Control, vol. 17, no. 6, pp. 3166-3174, December 2019, doi: 10.12928/TELKOMNIKA.v17i6.13119.

[23] L. Mendiboure, M. A. Chalouf, F. Krief, "Load-Aware and Mobility-Aware Flow Rules Management in Software Defined Vehicular Access Networks," IEEE Access, vol. 8, pp. 167411-167424, 2020, doi: 10.1109/ACCESS.2020.3023500.

[24] J. J. Moreno Escobar, et al., "Energy-Efficient Industrial Internet of Things Software-Defined Network by Means of the Peano Fractal," Sensors, vol. 20, no. 10, 2020, doi: 10.3390/s20102855.

[25] S. Toufga, et al., "Towards Dynamic Controller Placement in Software Defined Vehicular Networks," Sensors, vol. 20, no. 6, 2020, doi: 10.3390/s20061701. 